Harold A. McAlister and William I. Hartkopf (eds.)

\title{
IUE Studies of Hot Binary Stars
}

\author{
DAVID J. STICKLAND
}

Rutherford Appleton Laboratory, Didcot, OX11 0QX, England

\section{A MESSAGE FROM MY SPONSOR}

The International Ultraviolet Explorer (IUE) was launched on 26th January 1978 and is still fully operational today, with several more years hopefully to come. After six months, the fully-reduced data is consigned to public-access archives maintained by the project agencies (NASA, ESA, and SERC). Thus, in addition to observations from current and future programmes, there are $\sim 12,000$ high-dispersion $(\Delta \lambda \sim 0.15 \AA)$ spectra readily available now for research on stars hotter than about mid-B type. Furthermore, a uniform reprocessing, with optimum schemes, of all past IUE images has begun, to create the Final Archive which will be made accessible on-line through optical disk storage systems and will ensure the value of IUE data well into the future.

Just how IUE data can be used is a question bounded only by the imagination and ingenuity of the binary-star astronomer; for a flavour of the work done in the first ten years or so, see the review by Stickland (1988a). Of especial interest in the context of the present meeting, is the study of the orbital motion of hot stars. A summary of such work was presented to hot star enthusiasts in Bali in 1990 (Stickland 1991) but an update is now due both of the methods used to extract the radial velocities and of the areas of binary-star work where a useful contribution is being made.

\section{PER ARDUA...}

The operation of IUE in high-dispersion mode employs an echelle spectrograph which is fed by light through one of two apertures. Although the small aperture (diameter 3 arcsec) was frequently used in the early days when it was believed that it would yield better spectral resolution, observations are now almost always made using the large aperture (oval, $10 \times 20 \mathrm{arcsec}$ ) which hardly degrades resolution, permits shorter and reliable exposures, and allows photometry.

However, the on-board wavelength calibration lamps cannot sensibly be used with the large aperture, and so not only is no calibration spectrum imposed on the spectrum of the target but one cannot be secured on images taken immediately before or after the target star exposure either. Such wavelength calibration as is applied is done so on the basis of the dispersion constants derived from the small aperture with an appropriate geometrical shift. Given the possibilities of thermal or electronic shifts and the chance of less-than-perfect placement (or even deliberate off-centring) of the image in the aperture, a new approach is required to tie down the wavelength scale.

For hot stars, rescue comes in the form of the rich spectrum of interstellar (i/s) lines to be found in the far-and mid-ultraviolet spectral region: it requires but a modest column of gas to produce measurable features due to the resonance and other ground-state lines of cosmically abundant elements. One can generally 
identify at least 20 suitable $\mathrm{i} / \mathrm{s}$ lines in the short-wavelength (SWP) spectra ( $\lambda \lambda 1150-2000 \AA$ ) and a comparable number in the long-wavelength (LWP or LWR) spectra ( $\lambda \lambda 2000-3200 \AA)$. In modern data analysis systems, these lines can be measured (in an undefined reference frame) using some form of cursor setting or simple profile fitting. This approach was followed for the first eight papers in the series presently running in The Observatory, but it can become tedious when there are many spectra to deal with. Early failures to use crosscorrelation measurement on the assembly of interstellar lines, because of an inadequate treatment of the edge effects between the many small sections of spectrum containing i/s lines, have been overcome (Stickland et al. 1992) by the simple expedient of joining the sections of the rectified spectrum by 'padding' of constant value. The method has the added advantage of working when the $\mathrm{i} / \mathrm{s}$ line profiles are complex (asymmetrical or split).

Until Paper 8 of the series, measurement of photospheric lines was accomplished by cursor setting. An initial sweep was made through a sample of the target-star spectra and a large number of lines measured in each. Those that failed to give consistent results were jettisoned before the whole set of spectra were measured. The strong wind lines were excluded from measurement as were lines likely to be contaminated by $\mathrm{i} / \mathrm{s}$ components, leaving around 20 lines. This took a lot of time and, in cases where the lines were broad and noisy (the IUE $\mathrm{s} / \mathrm{n}$ is at best only 20 or so), it was inaccurate and frustrating. Ian Howarth saved the day with a cross-correlation routine in which the target spectrum could be compared with a mask derived from the same star (a single spectrum or an averaged one) or one of a star of similar spectral type, preferably with sharp lines. This was first used on V861 Sco (Stickland \& Howarth 1991) where the position of the peak of the cross-correlation was determined by parabola fitting. LZ Cep (Howarth et al. 1991) proved a bigger challenge since the spectra of both components could be seen and recourse was made to fitting a pair of Gaussians to the cross-correlation function, the widths and relative strengths of the individual components being fixed on the basis of well-separated spectra.

Thus cross-correlation on both $\mathrm{i} / \mathrm{s}$ and stellar lines represents the present development and is demonstrated in a paper on Y Cyg (Stickland et al. 1992). This work also shows that if, from, say, optical spectra, the absolute velocity of the interstellar lines is known for both target and standard, and the radial velocity of the standard used for the mask is also known (as it is for $\tau$ Sco which was used with Y Cyg), then absolute velocities can be obtained for the target.

If it is necessary to rely solely on UV measurements, the wavelengths of a sufficiency of features in the spectra of a range of spectral types will be required. This matter has not yet been adequately addressed although partial lists for a variety of objects are scattered through the literature. As a small step along the way, a number of reliable, $\mathrm{i} / \mathrm{s}-\mathrm{free}$, symmetric features in the spectrum of $\zeta$ Cas (B2 IV) have been measured on 12 SWP spectra and assigned vacuum wavelengths. Further line lists are being worked upon and one for the $09 \mathrm{Ib}$ star, HD 209975, is nearing completion. They are available on request. 


\section{3. ...AD ASTRA}

So where can IUE data benefit orbital studies of binary stars? The first paper considered $\iota$ Ori, where observations in the UV render the blending effects of the secondary component less problematical than in the optical (Stickland 1987). This leads naturally to composite spectra where one might want to escape contamination or domination by a cooler companion by fleeing to the UV. Stickland (1988a) discussed the particularly important case of Cepheid binaries but the results so far are tentative since the hot companions are generally of too late a type, such that the $\mathrm{i} / \mathrm{s}$ lines are plagued with contamination. However, valuable work has been done by Böhm-Vitense (1986) in using cross-correlation on both hot and cool components in S Mus.

One problem of working on early-type stars in the optical region is the paucity of lines suitable for consistent radial velocity measurement, leading often to rather poor orbital parameters. Several examples, such as $\delta$ Ori (Harvey et al. 1987), show the merits of using the rich UV spectra of O-type stars to beat down the rms residuals in orbit determination, although it has to be said that the recent application of cross-correlation methods to digitized optical spectra by Graham Hill and co-workers has greatly improved matters.

Fashion has pushed the determination of orbits down the list of priorities in many major observatories and, together with the 'one night stand' kind of time-allocation, complete coverage of orbital cycles becomes a tall order. This is where easy access to a large archive of reduced data comes in, and there is general agreement that IUE has set the pace here. Thus we have examples of stars which have been observed often, perhaps to study wind variability, that can now be examined for radial velocity variation. Sometimes, such as with $\mathrm{X}$ Per (Stickland 1992), one draws a blank. On other occasions one gets a modern orbit for comparison with earlier work to look for period changes (see, e.g., AO Cas (Stickland 1988b)) or apsidal motion ( $\delta$ Ori and Y Cyg).

So when you get back home, check the IUE log for your favourite stars, and if they are not there, apply for some time in the very next round. IUE will not last forever!

\section{REFERENCES}

Böhm-Vitense, E. 1986, ApJ, 303, 262

Harvey, A.S., Howarth, I. D., \& Zuiderwijk, E. J. 1987, The Observatory, 107, 205

Howarth, I.D. et al. 1991, The Observatory, 111, 167

Stickland, D.J. 1987, The Observatory, 107, 5

Stickland, D.J. 1988a, in ESA SP-281, 2, p27

Stickland, D.J. 1988b, The Observatory, 108, 174

Stickland, D.J. 1991, IAU Sym., 143, 237

Stickland, D.J. 1992, MNRAS, submitted

Stickland, D.J. \& Howarth, I.D. 1991, The Observatory, 111, 23

Stickland, D.J. et al. 1992, The Observatory, 112, in press 